\title{
Meter el dedo en la llaga: la fragilidad en el baile flamenco
}

\author{
To put a finger on the wound: the fragility in flamenco dance
}

\author{
FERNANDO LÓPEZ RODRÍGUEZ*
}

Resumen: en este trabajo parto de una presentación general del binarismo de las categorías de género, a la luz de una posible comprensión del mismo desde la axiología, para pasar, en un segundo momento, a analizar cómo se traducen dichas categorías en el cuerpo, a través de ciertas prácticas concretas. Asumiendo como metodología pertinente para la problematización de los fenómenos corporales el modelo de una «lectura cercana»**, escojo mi experiencia en primera persona como bailaor de flamenco para hacer ver cómo se produce la encarnación de las categorías de género en esta actividad concreta y qué estrategias de emancipación gestual resultan posibles. Palabras clave: baile flamenco, gesto, género, masculino, femenino.

\begin{abstract}
This work starts with a general introduction to gender categories, showed through a possible comprehension given by the axiology. In a second part, I analyze how these categories are translated into the body, through different practices. I assume the «close reading» method as the pertinent methodology to investigate the body phenomena and I choose my own experience as flamenco dancer to demonstrate how the embodiment of gender categories in this activity is produced and which strategies are possible to generate a gestural emancipation.
\end{abstract}

Keywords: flamenco dance, gesture, gender, masculine, feminine.

\section{Introducción}

La cuestión de género constituye, de un tiempo a esta parte, una suerte de cuarta dimensión de la «filosofía de la sospecha»: sospecha hacia la dicotomía fundamental establecida por el sexo y el género, tanto en el binarismo social y su reparto de roles, como en su traducción en el campo de la estética a «imagen y semejanza» del mundo que proyecta.

Fecha de recepción: 08/04/2016. Fecha de aceptación: 27/07/2016.

* Doctorando en el «Laboratoire Pratiques et Discours en Danse», en la Universidad París VIII. Sus ejes principales de investigación conciernen la filosofía de la danza, la antropología y estética del baile flamenco, el análisis de obras coreográficas y de procesos de composición y los estudios de género aplicados al análisis de las artes escénicas. Destaca su reciente trabajo galardonado con el I Premio de Investigación en Danza de la Academia de las Artes Escénicas de España «Espejismos de la identidad coreográfica: estética y transformaciones de la farruca», publicado en la colección Los libros de la Academia (2015), y su libro «De puertas para adentro: disidencia sexual y disconformidad de género en el baile flamenco» (Egales, 2016). Contacto: 1rparra.fernando@gmail.com

** Ginot, I. y Roquet, C. «Une structure opaque: les « Accumulations » de Trisha Brown», en: C. Rousier (ed.): $\hat{E}$ tre ensemble. Figures de la communauté en danse depuis le XXème siècle. Pantin, Centre National de la Danse, 2003, p. 265. 
Habremos de enfrentarnos, pues, a dos problemas fundamentales e internamente relacionados. En primer lugar, a la axiología subyacente al binarismo de género. En segundo lugar, a las formas de resistencia política favorables a una lucha contra el mismo, que deben de ser analizadas, por rigor metodológico, caso por caso, razón por la cual nos centraremos en su análisis dentro de la manifestación específica del baile flamenco, menos por interés intelectual de aficionado que por la urgencia práctica a la que me remite mi propia realidad cotidiana como trabajador de la danza en este ámbito.

\section{Género y axiología}

El problema fundamental del binarismo de género radica, no sólo en la forma parcelaria de entender la realidad, de la cual parece que los seres humanos somos deudores en gran medida debido a una cierta necesidad de simplificación de la heterogeneidad de los fenómenos, sino principalmente en que cada una de las categorías, tanto de sexo (hombre-mujer) como de género (masculino-femenino), no son sino la tapadera de la distinción entre valor positivo y valor negativo o disvalor.

El conjunto de características a las que se asocia lo masculino será valorizado positivamente, mientras que todas aquéllas que pertenecen al conjunto de lo femenino, serán consideradas como un disvalor o valor negativo.

Sin embargo, esto es todavía «demasiado sencillo para ser verdad», por retomar la optimista expresión de Heidegger: el binarismo de género no sólo divide la realidad en territorios de oposición, sino que asigna a los individuos, de manera aristotélica, un «lugar natural» de pertenencia en función de su sexo ${ }^{1}$.

Una persona de sexo mujer pertenece al género femenino, considerado como inferior, lo cual le concede un valor negativo. Sin embargo, si se corresponde a los estándares marcados por el mismo, le será conferido un valor positivo, en la medida en que permanece dentro de los cánones marcados para su sexo. Esta especie de «valor añadido» no contrarresta el disvalor asignado a la mujer; más aún: genera en la persona, en virtud de su adecuación al canon social, la ilusión de ser bueno, valioso, etc., lo cual imposibilita su emancipación en función del beneficio secundario que recibe, haciéndole creer (sea ésta una creencia verdadera o no) que dicha correspondencia es, en el fondo, «lo que verdaderamente quiere».

La hipótesis que planteo aquí es que es precisamente en virtud de dicho beneficio secundario que la creencia acerca de la correspondencia entre la propia volición y el estándar social se torna verdadera.

Esto quiere decir que el inmovilismo social prescribe una actitud de sumisión respecto de los roles definidos por las categorías de género, lo cual hace imposible una actitud diferente a la del conformismo, el cual es, a su vez, premiado y traducido en el sentimiento de estar cumpliendo con el deber, de ser una «buena mujer». No cumplir con el deber provoca, por lo tanto, un sentimiento de culpa.

1 Renunciamos a dar cuenta de la complejidad del fenómeno de la intersexualidad y la transexualidad por honestidad intelectual ante el escaso conocimiento que poseemos de los mismos y por la falta de presencia (hasta donde llega nuestro conocimiento) en el ámbito del baile flamenco, que es el que nos proponemos analizar. 
En el caso de los hombres, la cuestión varía ligeramente: al renunciar a los privilegios concedidos por su pertenencia al grupo dominante, la emoción que predomina es la de la vergüenza por la pérdida de poder que esto supone, es decir, por el hecho de «dejarse dominar».

\section{Conmigo o contra mí: cambiarse de acera}

A pesar de todo lo dicho hasta ahora, el pensamiento dicotómico funciona de tal modo que siempre acaba encontrando maneras de catalogar las prácticas de los seres humanos, de manera que todo en la realidad puede variar excepto las categorías que aplicamos para la explicación de los hechos.

Es, de esta manera, que el amigo pasa a ser identificado como enemigo cuando se cambia de bando (o de acera) entendiendo que dicha elección es el resultado de algún tipo de trastorno psiquiátrico o de un mal acoplamiento entre el género del cuerpo (por ejemplo, masculino) y el género del alma que éste encarna (femenino). Históricamente, como bien es sabido, la creatividad humana se ha ejercitado en encontrar toda una gama de variopintas justificaciones.

Sin embargo, el tercio excluso no hace a veces sino reproducir el binarismo del principio de no contradicción intentando hacer plausible la existencia de excepciones, como una suerte de ampolla que se abriera entre una cosa y su contraria, dilatando ligeramente el espacio de lo posible.

Podríamos decir que, en nuestro relato, el orden social ha ido salvando los peligros de su propia inmolación hasta el momento, buscando los subterfugios necesarios para razonar la solidez de sus categorías.

A pesar de todo ello, existe un último fenómeno que se resiste a su fijación, que impide el funcionamiento cuerdo de las oposiciones binarias y que se efectúa como un tránsito permanente entre los dos grupos. El amigo es enemigo y el enemigo es amigo, o lo que es lo mismo: el diferente es como yo y el compadre se comporta como el diferente porque renuncia a la fijación identitaria.

Esto es parte de lo que podría llamarse queer: no la versatilidad o la capacidad de aparecerse en una y otra dimensión del género, sino la permanente transición entre ambos, o lo que sería más preciso: el habitar una posición de exterioridad a ambos, generando un discurso de inconformismo que permite la elaboración constante de nuevas prácticas, en función de la combinatoria potencialmente infinita de rasgos atribuidos a uno u otro conjunto.

Lo valorado positivamente, ahora, no será la pertenencia a uno u otro sino la capacidad de elaborar un discurso propio, potencialmente abierto a la transformación, que no desarrolle un vínculo de identificación o de fijación con ningún bloque de características u opciones.

Efectivamente, la «identidad líquida», tan simpática para el capitalismo, suena próxima a la cuestión queer, y sin embargo difiere enormemente. La identidad líquida del capitalismo se articula sin eje ni principio regulador en función de una variabilidad de caracteres que se van modificando en exclusiva relación con el mecanismo del deseo. Existe, sin embargo, una doble identificación: por un lado, entre ese puro desear y el objeto (persona, acción o cosa) aleatoriamente escogido y por otro lado, y de manera aún más profunda, entre la persona deseante y el proceso mismo de desear. 
Lo queer respondería, tal vez, a la necesidad de no identificarse ni con el mecanismo del deseo ni con su objeto porque sabe (porque lo ha vivido) que los procesos identitarios no se elaboran sin una pareja exclusión del diferente y sin un consecuente establecimiento de jerarquías de poder.

\section{Las trampas del inmovilismo categorial}

Llegados a este punto, habría que hablar de qué es lo que sucede más precisamente en el arte de la escena con las categorías sociales en su relación con el público, porque las opciones son escasas: o bien reproducimos, sin ninguna brecha crítica, los modelos sociales (de tal modo que lo ocurrido en escena no sería sino un reflejo complaciente de un público que busca reconocerse y regocijarse con la representación); o bien introducimos le ver dans le fruit (el gusano en la manzana), siguiendo la expresión utilizada por Isabelle Launay ${ }^{2}$, para hacer un arte que no «señala con el dedo» aquellas zonas de la realidad cuya negatividad trata de evidenciar sino que «pone el dedo en la herida».

Cada forma artística deberá buscar sus propios recursos y estrategias para evitar las dos trampas del inmovilismo categorial, al cual pertenece el inmovilismo generado por el binarismo de género.

Primeramente, la reproducción de las mismas jerarquías de poder en su actividad cotidiana: no sólo cuando el espectador está mirando sino «de puertas para adentro», lo cual implica interrogarse continuamente sobre el tipo de relaciones que los artistas mantenemos con nosotros mismos y con las personas con las que nos rodeamos.

Un claro ejemplo de esto, en el mundo de la danza, es el tipo de relación que el coreógrafo establece con sus bailarines. El modelo clásico del coreógrafo demiurgo establecía una comunicación vertical y unívoca de él hacia los bailarines intérpretes, quienes, además de permanecer exentos de voz y voto en el proceso creativo, recibían en su cuerpo la obra coreográfica por una suerte de emanación y se plegaban a ella sin que la particularidad de sus cuerpos pudiera interferir en el devenir-concreto de la idea, y mucho menos modificarlo. Mantenerse vigilante acerca de este tipo de relaciones, siendo ésta nada más que un ejemplo entre otras muchas, resulta de vital importancia.

La segunda trampa que cada forma artística deberá tratar de evitar es la de la complacencia con el público. Por intereses económicos o por instinto maternal, los artistas tratamos a menudo de ahuyentar la negatividad (que poco o nada tiene que ver con hacer al espectador sentirse culpable, como bien sobre ello ha reflexionado Jacques Rancière ${ }^{3}$ ) devolviéndole a la sociedad una imagen inmaculada de sí misma, que no sólo aparece en formas artísticas de carácter naíf, sino en aquéllas en las que la negatividad (traducida a menudo en provocación vacía) constituye una especie de bálsamo para una clase media atormentada por su incapacidad, real o imaginaria, de cambiar las cosas. Asistir a esta clase de actos artísticos le devuelve una imagen lavada de sí misma, expiando así el pecado y permitiéndole continuar con su vida evacuando la posibilidad de transformación.

2 «Gestes tordus, gestes toxiques, gestes revenants sous le signe des grimaces de la Venus hottentote de Jane Avril et de Joséphine Baker. De Self Portrait Camouflage (2006) à Adieu et merci (2014)», en: A. Baudelot (ed.) : Grimaces du réel. Latifa Laâbissi. Paris, Les presses du réel, 2016, pp. 34-64.

3 «Les paradoxes de l'art politique». En Le spectateur émancipé. La fabrique éditions. París, 2008. 
Evitar la segunda trampa quiere decir, por tanto, aprender a gestionar, al modo alquimista, la cantidad y la vibración de negatividad necesaria para que una experiencia crítica se despierte en el cuerpo del espectador: sensitiva, emocional o intelectualmente.

\section{Desmontar categorías en el cuerpo}

Nuestros problemas son siempre concretos. Por eso, después de haber trazado este paisaje general, me parece necesario acotar el terreno de estudio para entender bien aquello de lo que estamos hablando: no del arte en general sino de las artes escénicas; más aún, de la danza; más aún, del baile flamenco. Y dentro del baile flamenco, la problemática que nos ocupa, que no es lógicamente independiente de muchas otras y que nos puede servir para entrever muchas otras: la división genérica (baile de hombre, baile de mujer).

Nuestra metodología consistirá, como hemos anunciado, en meter el dedo en la llaga. Poner el dedo en la herida es una cuestión de sensibilidad. De trabajar con las sensaciones del cuerpo para distorsionar las fronteras de la costumbre comúnmente compartida. Para cuestionar el sentido común, o la comunidad de los sentidos, es decir, el hecho de que todos sintamos lo mismo en una determinada situación y pensemos, por tanto, lo mismo, convergiendo en esa universalidad subjetiva deseada por Kant en el $\$ 5$ de su Crítica de la facultad de juzgar.

En el caso del flamenco poner el dedo en la llaga es un acto sutil y complejo que toca en lo más profundo de su estructura, y que, respecto de la cuestión de género, poco o nada tiene que ver con el travestismo, más o menos parcial, de intérpretes cuyos cuerpos no se transforman bajo la ropa.

Meter el dedo en la llaga del flamenco quiere decir hacerle vivir su propia fragilidad, más allá de la dignidad o la indignación del llanto, el grito y la histeria, individual o colectiva. Más allá de la poética de la hipertonía, la fragilidad. Un flamenco débil, por una vez. Recién despierto o todavía medio dormido. O enfermo, casi sin fuerzas. Moribundo. Lejos del drama del escarnio público. Un flamenco cansado de resistir. Es decir, un flamenco que trabaja desde una tonicidad más baja que le permite crear una acuarela mayor de acentuaciones en el propio movimiento, y que inventa un nuevo vocabulario de acciones posibles en el que ya no sólo se golpea, se marca, se llama o se remata, sino que también se tropieza, se pierde, se marea, se desequilibra, se cae, lo intenta, tiembla, pierde, se cansa, se agota, no termina, se rinde.

En una de mis tesinas ${ }^{4}$ exploré en detalle la cuestión del flamenco como una «estética de resistencia» que hacía a hombres y mujeres, más allá del catálogo de diferencias establecidas por la diferenciación de género, iguales ante una misma ley: la ley del peso.

Siguiendo la caracterización de los factores del movimiento de Rudolf Laban en su teoría del $e f f o r t^{5}$, el peso oscilaría entre dos polos extremos: el fuerte y el ligero. Intuitivamente asignamos al peso fuerte una vinculación con el tren inferior del cuerpo y con la relación de anclaje al suelo, mientras que lo ligero es asimilado al desapego del mismo y, con ello, a las estéticas de la elevación.

4 Mâl(e) d'archive. Vers une histoire queer de la farruca. Departamento de Danza de la Universidad Paris VIIIVincennes-Saint Denis. Dirigida por Isabelle Launay. Defendida en junio de 2015.

5 Los factores del movimiento según Laban son tiempo, espacio, flujo y peso. Cada uno de ellos posee dos polos opuestos que son, sostenido o repentino para el tiempo, directo-focal o indirecto-foveal para el espacio, condensado o libre para el flujo y fuerte o débil para el peso. 
De todas las combinatorias posibles entre los factores de movimiento propuestos por Laban, sólo el peso ligero aparece como lugar prohibido para el flamenco: visitarlo es encontrar una puerta de salida a su estética, al entenderlo, de nuevo, como algo que va más allá de la elevación y lo volátil, y que podría tener que ver con la fragilidad, en sentido físico y psicológico.

Es por ello que incluso el modelo de mujer flamenca es el de una mujer fuerte o fálica, según cierta terminología de origen psicoanalítico, que encarna un gran número de valores atribuidos a la masculinidad y cuya feminidad se traduce, casi exclusivamente, en aceptar ser objeto de seducción para la mirada masculina (razón fundadora del uso permanente de accesorios, tanto de mero adorno -flores, peinetas, volantes, etc. - como de «apéndice corporal» para el cuerpo -abanico, mantón, bata de cola...-).

Por lo tanto, el trabajo de la fragilidad no sería una necesidad exclusiva del hombre sino una ejercitación quinestésica de la que también habría de ser partícipe la mujer.

Asimismo, las formas de feminización del flamenco por parte de los hombres no serán efectivas si pasan exclusivamente por el uso de accesorios de mujer sin renunciar al subterfugio del control del propio movimiento y de la imagen del sí-mismo: la bata de cola, el abanico o el mantón son dispositivos neutros que no fragilizan la calidad del movimiento si el uso que hacemos de ellos sigue efectuándose desde el mismo espectro de coloración gestual que excluye el peso débil. ${ }^{6}$

Formas de travestismo o de pseudotravestismo, como el reciente caso de Manuel Liñán bailando unos Caracoles con bata de cola y mantón en el espectáculo Los invitados de Belén Maya (y posteriormente en sus producciones Nómada y Reversible -donde por cierto, la cuestión de género es presentada como tema central de la pieza-) resultan revoluciones políticas abortadas, dado los valores masculinos que presiden toda la coreografía: virtuosismo técnico, velocidad, hipertonía, peso fuerte...

No es baladí que esta acción, acaecida por primera vez en el Festival de Jerez de 2014, tuviera como continuidad la institucionalización de la misma (y, por tanto, desde mi punto de vista, la desactivación de su potencial generador de cambio) a través de la aparición de la imagen del citado bailaor, usando dichos accesorios, como cartel oficial de la edición del mismo festival al año siguiente.

Es por ello que los casos más cercanos a este trabajo del peso ligero que hemos encontrado han sido en viejos y en amateurs. Los viejos, como podemos observar en documentales como Triana, pura y pura $^{7}$, bailan con una tonicidad más baja, con menos intensidad, sustrayéndose a lo que he dado en llamar el «subrayado tónico», que tiene que ver con la tendencia constante a dar más intensidad a un movimiento para concederle más importancia y/o hacerlo más visible, y que conduce en el flamenco, a veces, a un estado hipertónico

6 Sobre el uso de la sensualidad y la mímesis del acto sexual por parte de los bailaores sería necesario abrir un amplio capítulo nuevo en el que habría de diferenciarse el carácter sexual de ciertos bailes (que permiten al bailaor hacer uso de «zonas prohibidas» de su cuerpo -como las caderas-), como es el caso de los tangos y las bulerías (contexto festivo), del ser-objeto sexual para la mirada del otro.

En primer lugar, porque nunca he sido espectador de dicha mímesis ejecutada fuera del marco de una relación heterosexual, razón por la cual la gestualidad del hombre es la propia de aquél que ejecuta el rol activo. En segundo lugar, porque la seducción en el hombre, para adquirir esa tonalidad afeminada, debería vehicular el imaginario de un intérprete dispuesto a no ser aquél que domina en el acto sexual, es decir, de un intérprete dispuesto a ser pasivo y, por tanto, la manera de ofrecer su cuerpo a la mirada del otro habría de ser totalmente otra.

7 Ricardo Pachón. Universal, 2013. 
constante sin matices, que se traduce en una estética histérica que siempre nos hace recordar ese «exceso de presencia» con el que Gilles Deleuze describe la pintura de Francis Bacon ${ }^{8}$.

El caso de los amateurs, en algún sentido más complejo y por tanto imposible de agotar aquí en toda su diversidad, tiene que ver con la torpeza en el gesto: estado de confusión, de gesto «sucio» o no finalizado, de desequilibrio postural, de titubeo en la ocupación del espacio o de falta de acorde en la relación con la música. En todos los casos, lo que se hace visible es la fragilidad de alguien mostrándose en una situación que no controla completamente.

En el flamenco el tránsito posible entre los dos roles de género puede adoptar muchas estrategias pero adquiere un común denominador: el peso ligero como fragilidad, lo cual implica una renuncia al control total del movimiento y de la imagen que de éste percibe el espectador. Una renuncia también, por tanto, a la danza como estética meramente formal.

Es ahí donde encontramos el límite entre el flamenco y su exterioridad, el punto en el que una determinada estética está a punto de bascular hacia otro lado, no para convertirse en otro lenguaje coreográfico reconocible sino, tal vez, para permanecer en ese estado que podríamos calificar como inconformista, o como queer.

Poner el dedo en la herida, por tanto, sería un método para desmontar, desde el cuerpo, binarismos categoriales vigentes en el mundo social y en el mundo del arte, pasando por la sensación, preguntándonos: ¿qué no me es permitido sentir y de qué manera puedo reencontrar esa sensación?

$\mathrm{Si}$, como hemos afirmado anteriormente, «todos nuestros problemas son concretos», entonces la solución a los mismos habrá de serlo también, y todo el proyecto postural y el abanico de gestos fagocitado inconscientemente por el cuerpo (lo que el sociólogo francés Bourdieu condensa en la noción de hábitus), teñidos ambos de esa normatividad social, habrán de ser disueltos desde la concreción del cuerpo y el trabajo de la sensación.

\section{Bibliografía}

Cleminson, Richard y Vázquez García, Francisco (2011): Los invisibles. Una historia de la homosexualidad masculina en España, 1860-1939. Editorial Comares, Granada.

Godard, Hubert (1992): «Présentation d'un modèle de lecture du corps en danse», en : M. Arguel, Le corps en jeu. PUF, París.

Godard, Hubert (1997): «Le poids des trans-actions», en Age du corps, maturité en danse (actes de colloques). La cratère d'Alès. Scène Nationale, París.

Godard, Hubert (2006): «Des trous noirs. Entretien avec Hubert Godard par P. Kuypers», en : I. Corin (ed.) : Nouvelles de Danse. Contredanse, 53, Bruselas.

Godard, Hubert (2008): «Le geste et sa perception», en : I. Ginot, \& M. Michel (eds.) : La danse au XXe siècle. Larousse, París.

Heffner Hayer, Michelle (2009): Flamenco. Conflicting Histories of the dance. McFarland\& Company, Londres.

Mira, Alberto (2007): De Sodoma a Chueca. Una historia cultural de la homosexualidad en España en el siglo XX. Ediciones Egales, Barcelona.

Vidarte, Paco (2007): Ética marica. Editorial Egales, Madrid.

8 Deleuze, G. Francis Bacon. Logique de la sensation. París, Editions du Seuil, 2002. 
